\title{
Nucleosynthesis of light trans-Fe isotopes in ccSNe: Implications from presolar SiC-X grains
}

\author{
Waheed Akram ${ }^{1, *}$, Khalil Farouqi ${ }^{2}$, Oliver Hallmann ${ }^{2,3}$, and Karl-Ludwig Kratz ${ }^{2, *}$ \\ ${ }^{1}$ Department of Earth Sciences, University of Oxford, Oxford OX1 3AN, UK \\ ${ }^{2}$ Department of Chemistry, Pharmacy \& Geosciences, Univ. Mainz, D-55128 Mainz, Germany \\ ${ }^{3}$ Max-Planck Institute for Chemistry, Otto-Hahn Institute, D-55020 Mainz, Germany
}

\begin{abstract}
This contribution presents an extension of our r-process parameter study within the high-entropy-wind (HEW) scenario of corecollapse supernovae $(\mathrm{ccSNe})$. One of the primary aims of this study was to obtain indications for the production of classical p-, s- and r-isotopes of the light trans-Fe elements in the Solar System (S.S.). Here, we focus on the nucleosynthesis origin of the anomalous isotopic compositions of $\mathrm{Zr}, \mathrm{Mo}$ and $\mathrm{Ru}$ in presolar $\mathrm{SiC} \mathrm{X}$-grains ( $\mathrm{SNe}$ grains). In contrast to the interpretation of other groups, we show that these grains do not represent the signatures of a 'clean' stellar scenario, but rather, are mixtures of an exotic nucleosynthesis component and S.S. material. We further confirm the results of our earlier studies whereby sizeable amounts of all stable p-, s- and r-isotopes of $\mathrm{Zr}$, Mo and $\mathrm{Ru}$ can be co-produced by moderately neutron-rich ejecta of the low-entropy, charged-particle scenario of ccSNe (type II). The synthesis of these isotopes through a 'primary' production mode provides further means to revise the abundance estimates of the light trans-Fe elements from so far favoured 'secondary' scenarios like Type Ia $\mathrm{SNe}$ or neutron-bursts in exploding massive stars.
\end{abstract}

\section{Introduction}

The nucleosynthetic origin of the stable isotopes in the light trans-Fe region between $\mathrm{Zn}(\mathrm{Z}$ = 30) and $\mathrm{Ru}(\mathrm{Z}=44)$ in the Solar System (S.S.) has been a fascinating subject for astrophysicists, astronomers, nuclear chemists and physicists, and cosmochemists for over 60 years [1 - 3]. In this multidisciplinary community, it is commonly believed that these elements are produced by various contributions from three different historical stellar processes: (i) the 'p-process' [4, 5], (ii) the 'weak s-process' ([6, 7] and refs. therein), and (iii) the 'weak r-process' ([8 - 10] and refs. therein).

Apart from the bulk S.S. isotopic abundances [11], more recent astronomical observations of elemental abundances in metal-poor halo stars [12 - 18] have revived and intensified the general interest in the nucleosynthesis of these elements beyond $\mathrm{Fe}$ and have motivated various theoretical studies with increasing realism (see extensive references in [19]). Supplementing these studies are isotopic analyses of meteoritic inclusions such as

*Corresponding author(s): waheed.m.akram@gmail.com., klk@uni-mainz.de 
presolar grains and $\mathrm{Ca}, \mathrm{Al}$ rich inclusions (see e.g. [20 - 24]). In particular, the interpretation of the anomalous $\mathrm{Zr}$, Mo and $\mathrm{Ru}$ isotope compositions of $\mathrm{SiC}-\mathrm{X}$ grains have so far defied a straightforward p-, s- or r-process explanation, requiring alternate nucleosynthesis scenarios. One such favoured process for the production of these light trans-Fe elements is the 'secondary' (i.e. metallicity dependant) neutron-burst (n-burst) occurring in the shocked He-shell of exploding massive stars [25 - 27]. Each of these models considered so far, however, are unable to co-synthesise all light $\mathrm{p}-$, s- and r-process isotopes in S.S. proportions [11], and struggle even more so in reproducing the abundance ratio of the two most abundant p-isotopes ${ }^{92} \mathrm{Mo}$ and ${ }^{94} \mathrm{Mo}$.

We offer a solution to these problems by following up on our earlier preliminary results $[28,29]$ exploring the 'primary' production of the light trans-Fe elements in a classical core-collapse supernova (ccSN), low-entropy, neutrino-driven-wind scenario.

\section{Theory: Parametric core-collapse supernova HEW model}

The notion of a high-entropy-wind (HEW), or neutrino-driven-wind (NDW), arises from considerations of the newly born proto-neutron star in ccSNe. In this scenario neutrinos interact with matter of the outermost neutron-star layers, leading to proton-rich and moderately neutron-rich HEW ejecta, with initially high entropies [30, 31]. In this present paper, as in our earlier publications [28, 32, 33], the nucleosynthesis calculations up to the charged-particle $(\mathrm{CP})$ freezeout were performed with continuously extended versions of the Basel network code (see [34]), following the description of adiabatically expanding homogeneous mass zones [35].

After CP $(\alpha)$ freezeout, the expanding (and eventually ejected) mass zones from the outer neutron-star layers have different initial entropies $\left(S \approx T^{3} / \varrho\left[\mathrm{k}_{\mathrm{B}} /\right.\right.$ baryon]; $\mathrm{T}$ : temperature, $\varrho$ : matter density) so that the overall explosion represents a superposition of different entropy 'intervals', correlated with (1) different electron abundances $\left(Y_{e}=Z / A\right)$, (2) different ratios of free neutrons to 'seed nuclei' $\left(Y_{n} / Y_{\text {seed }}\right)$ for the ensuing r-process, and eventually also with (3) different expansion velocities $\left(V_{\text {exp }}\right)$, which determine the respective durations of the $\alpha$-freezeout $\left(\tau_{\alpha}\right)$ and the r-process $\left(\tau_{r}\right)$. With the assumption that each equidistant entropy interval contributes an equal amount of ejected matter (see [36] for details), the above correlations within our HEW model can be expressed by a simple ' $\mathrm{r}$ process strength function', $Y_{n} / Y_{\text {seed }} \approx V_{\text {exp }} \times\left(S / Y_{e}\right)^{3}$. Using this parameterised approach, our HEW model is able to reproduce the astronomical and cosmochemical observations of different r-process 'components' using different $S$ and $Y_{n} / Y_{\text {seed }}$ ranges. The sum total of this multiplicity of r-process types has resulted in our complex S.S. r-process 'blend' composition.

Taking $Y_{e}=0.45$ to represent a typical case of a moderately neutron-rich ejecta, the lowest entropy range $(S \leq 50)$ represents the normal, rapid $\alpha$-rich freezeout without free neutrons $\left(Y_{n} / Y_{\text {seed }} \approx 10^{-14}\right)$, with a primary production of mainly stable or close to stable isotopes in the region of $\mathrm{Fe}$ to $\mathrm{Sr}$ (see second column of Table 1 in [32]). Moving onto a higher S-range $\left(50<S \leq 100\right.$; see column 3 of Table 1 in [32], with $Y_{n} / Y_{\text {seed }} \approx 1$ for $\left.S=100\right)$ there are not yet enough free neutrons available to start a real neutron-capture (r-) process. However, under these $S$-conditions at freezeout, the seed composition is already shifted to the neutron-rich side of $\beta$-stability, including the well-known $\beta$-delayed neutron isotopes in the $80<A<100$ mass region. In the next higher $S$-range $(100<S \leq 150)$ of our HEW model, the density of free neutrons $\left(1 \leq Y_{n} / Y_{\text {seed }} \leq 13\right)$ becomes high enough to start a 'weak' r-process up to the rising wing of the $A \approx 130$ S.S.-r $\mathrm{N}_{\odot}$ peak. Finally, for high entropies of $150<S \leq 280$, now with $Y_{n} / Y_{\text {seed }} \approx 155$, our HEW parameter approach predicts quite a robust 'main' r-process starting with the $N=82$ r-progenitor isotopes of ${ }^{43} \mathrm{Tc}$ to ${ }^{45} \mathrm{Rh}$ 
at the onset of the $A \approx 130$ peak extending beyond the $\mathrm{N}_{\odot, r}$ peak at the $N=126$ shell closure up to the actinide region.

Initially, the HEW model was successfully applied to the light trans-Fe elemental abundance patterns of metal-poor halo stars $[32,34]$ and later the S.S. isotopic abundances [28] whereby we discussed for the first time the possibility of a primary co-production of all p-, s- and r-process isotopes between ${ }^{64} \mathrm{Zn}$ and ${ }^{104} \mathrm{Ru}$ within the low-S, CP component of the HEW. As an example, the co-production of all 7 Mo isotopes over different $S$ intervals is illustrated in Fig. 1. Now we focus our attention on a third set of observations: the anomalous isotopic pattern of the three neighbouring even- $\mathrm{Z}$ elements $\mathrm{Zr}$, Mo and $\mathrm{Ru}$ in presolar silicon carbide X-grains [20, 21] building on our earlier work [19, 28, 37].
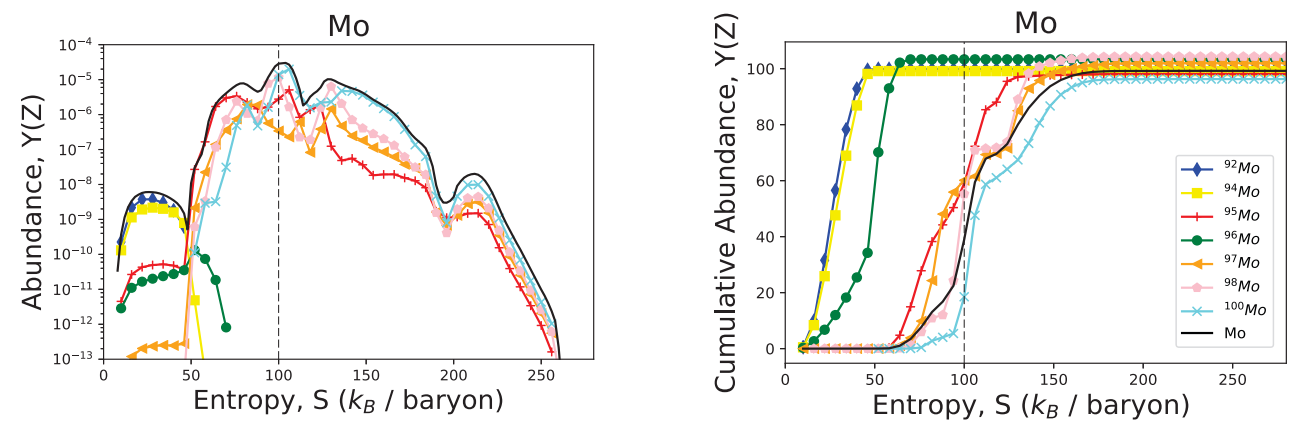

Fig. 1. Isotopic yields (left) and cumulative isotopic yields (right) for Mo as a function of cumulative entropy bins, for the case $Y_{e}=0.45$. CP component $(\mathrm{S} \leq 100)$ and n-capture component $(\mathrm{S}>100)$.

\section{Observations: presolar silicon carbide-X grains}

Silicon carbide $(\mathrm{SiC})$ grains are one of the best studied presolar grain types (see $[38,39]$ and refs. therein). The majority of $\mathrm{SiC}$ grains (mainstream grains) have compositions (e.g. low ${ }^{12} \mathrm{C} /{ }^{13} \mathrm{C}$, high ${ }^{14} \mathrm{~N} /{ }^{15} \mathrm{~N}$ relative to S.S.) indicative of formation around thermally pulsating asymptotic giant branch (AGB) stars, whereas a rare subtype ( $\approx 1 \%$; Type-X grains) have isotopic signatures (elevated ${ }^{12} \mathrm{C},{ }^{15} \mathrm{~N},{ }^{28} \mathrm{Si}$ relative to S.S. and excesses of ${ }^{26} \mathrm{Mg},{ }^{44} \mathrm{Ca},{ }^{49} \mathrm{Ti}$ ) that are more characteristic of explosive scenarios such as ccSNe ([21], [26] and refs. therein). The light element (up to $\mathrm{Fe}$ ) compositions permit us to regard $\mathrm{SiC}-\mathrm{X}$ grains as supernova condensates (SUNOCONs; see [26] and refs. therein), but the light trans-Fe isotopic compositions of SiC-X grains have so far evaded a straightforward classical (p-, s-, r-) interpretation $([21,22])$, showing instead some agreement with the nburst model of $[25,26]$.

In this contribution we focus largely on Mo, due to the increased robustness of the Mo isotope data. Using the standard cosmochemical conventions (e.g. see [19]), the isotopic compositions of the grains $\left({ }^{i} x\right)^{k} x ; x=$ Mo) are reported as deviations from the S.S. composition $\left({ }^{i} x /^{k} x_{\odot}\right)$ in parts per thousand $\left(\delta^{i} x_{k}\right)$, where $i$ represents the target isotope and $k$ represents the normalisation isotope. The grains are characterised by a large range of isotopic deviations from S.S.: i.e. depletions in the classical $\mathrm{p}-\left({ }^{92,94} \mathrm{Mo}\right)$ and $\mathrm{r}-\left({ }^{100} \mathrm{Mo}\right)$ isotopes, enrichments in the largely s-isotopes $\left({ }^{95,98} \mathrm{Mo}\right)$, whereas the s-only isotope $\left({ }^{96} \mathrm{Mo}\right)$ is present in almost identical abundances as ${ }^{97} \mathrm{Mo}$ (the normalising isotope). As for $\mathrm{Zr}$, the $\mathrm{SiC}-\mathrm{X}$ grains are characterised by depletions relative to ${ }^{90} \mathrm{Zr}\left({ }^{\mathrm{i}} \mathrm{Zr} /{ }^{90} \mathrm{Zr} \leq 0.5, i=91,92,94\right.$, 96). The $\mathrm{Ru}$ isotope signatures in these grains are more complex and the analysis hindered as $\mathrm{Ru}$ isotope data are only available for two $\mathrm{SiC}-\mathrm{X}$ grains, with relatively large uncertainties. Regardless, we find that the lighter isotopes $\left({ }^{96,98,99,100} \mathrm{Ru}\right)$ are on average under-abundant relative to ${ }^{101} \mathrm{Ru}$, whereas ${ }^{104} \mathrm{Ru}$ is present in similar proportions as ${ }^{101} \mathrm{Ru}$, 
and ${ }^{102} \mathrm{Ru}$ is over-abundant relative to ${ }^{101} \mathrm{Ru}$. In all cases it is evident that the complex isotopic signature of these grains is not representative of a classical p-, s- or r-process.

Plotting the isotopic data in cosmochemical three-isotope space (e.g. ${ }^{i} \mathrm{Mo} /{ }^{k} \mathrm{Mo}-$ ${ }^{\mathrm{j}} \mathrm{Mo} /{ }^{\mathrm{k}} \mathrm{Mo}$; as explained in [19]), the SiC-X grains define a linear correlation for Mo and $\mathrm{Zr}$ isotopes (Fig. 2). These linear trends with the S.S. composition at one extreme and the SiC$\mathrm{X}$ grain B2-05 on the other end (see Fig. 2) are interpreted as mixing lines, which strongly suggest that $\mathrm{SiC}-\mathrm{X}$ grains are admixtures of two distinct nucleosynthetic components (or end members), as already recognised by [22]. Whereas the data support one end-member having a S.S. composition, we do not claim that the composition of B2-05 (other extreme of mixing line) reflects that of the second end-member, but rather B2-05 is the 'purest' grain with the cleanest signature of the $\mathrm{SiC}-\mathrm{X}$ grain source. Thus we argue that $\mathrm{SiC}-\mathrm{X}$ grains do not represent pure nucleosynthetic signatures from a single process but are best explained as a mixture of an exotic nucleosynthesis component - SiC-X end-member ( $\mathrm{SiC}-\mathrm{X}_{\mathrm{EM}}$; approximated by grain B2-05) - with homogenised stardust of S.S. composition. The isotopic compositions of grain B2-05 can thus be used to constrain the isotopic signatures of the stellar source where SiC-X grains formed (see second column, Table 1).
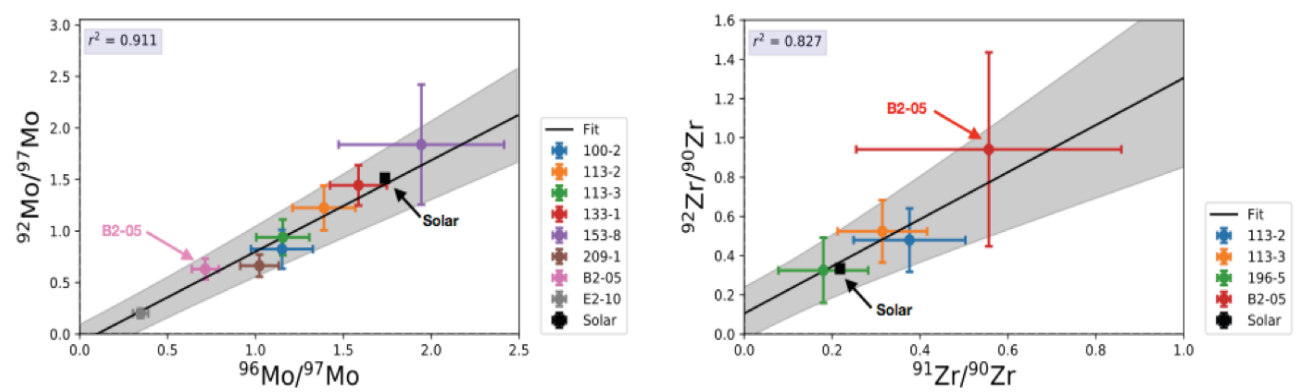

Fig. 2. Mo and $\mathrm{Zr}$ three-isotope plots for SiC-X grains ( $2 \sigma$ uncertainties). 2D data fit (line), associated $2 \sigma$ errorband (shaded area) and quality of fit $\left(r^{2}\right)$ also shown. See [19] for details on grain E2-10 (excluded from this analysis).

\section{Searching for the stellar source of SiC-X grains}

We now consider new and updated classical models (s-, r-, p-, n-burst) alongside our HEW scenario as potential candidates for the exotic $\mathrm{SiC}-\mathrm{X}$ end-member $\left(\mathrm{SiC}-\mathrm{X}_{\mathrm{EM}}\right)$. Selected isotopic ratios from these stellar sources (described below and in more detail in [19]) are given in Table 1, alongside the experimental constraints on the $\mathrm{SiC}-\mathrm{X}_{\mathrm{EM}}$ composition.

Classical and updated s-, $r$-, p-process: s-process models from [40] through to [41], and the diverse scenarios highlighted in the F.R.U.I.T.Y database [42], are all generally characterised by the absence of ${ }^{92} \mathrm{Mo}$, and high ${ }^{96} \mathrm{Mo} /{ }^{97} \mathrm{Mo}$ and ${ }^{98} \mathrm{Mo} /{ }^{97} \mathrm{Mo}$ relative to SiC$\mathrm{X}_{\mathrm{EM}}$, ruling out the s-process as the nucleosynthetic source of $\mathrm{SiC}-\mathrm{X}$ grains (see Fig. 3). rprocess yields are inferred using the r-residual method using the same aforementioned sprocess models [40 - 42]. The resulting r-process signatures are over-abundant in ${ }^{100} \mathrm{Mo}$ relative to SiC-X grains, and show no signs of ${ }^{92,94} \mathrm{Mo}$ (classical p-only) or ${ }^{96} \mathrm{Mo}$ (shielded from the r-process pathway by ${ }^{96} \mathrm{Zr}$ ) making the r-process an unlikely candidate. The improved yields (see [19]) of p-process nucleosynthesis in Type-Ia SNe [43] are preferred over earlier work on electron-capture SNe of AGB stars [44]. Despite the 'secondary' nature of this process, resulting from the use of an enhanced s-seed distribution in the progenitor AGB star, large excesses in the p-nuclides $\left({ }^{92,94} \mathrm{Mo}\right)$ and under-abundances of ${ }^{97}$ Mo relative to all other Mo isotopes allow us to eliminate p-process nucleosynthesis [43] as the source of $\mathrm{SiC}-\mathrm{X}$ grains. 
n-burst: as already discussed in [22] and shown in Table 1 and Fig. 3, the n-burst model [25] succeeds to some extent in explaining the anomalous Mo (and $\mathrm{Zr}$ ) isotope signatures in $\mathrm{SiC}-\mathrm{X}$ grains, and to a lesser extent $\mathrm{Ru}$ (where too little ${ }^{96,98} \mathrm{Ru}$ is synthesised). However we remind the reader that (1) the concordance for ${ }^{95} \mathrm{Mo} /{ }^{97} \mathrm{Mo}$ between the $\mathrm{n}$-burst model $[26,27]$ and data results solely from $\mathrm{SiC}-\mathrm{X}$ grains having a close to S.S. ${ }^{95} \mathrm{Mo} /{ }^{97} \mathrm{Mo}$ value and the n-burst model naturally predicting S.S. ${ }^{95} \mathrm{Mo} /{ }^{97} \mathrm{Mo}$ values due to its dependence on an initial S.S. seed composition, and (2) although the ${ }^{92} \mathrm{Mo} /{ }^{97} \mathrm{Mo}$ and ${ }^{94} \mathrm{Mo} /{ }^{97} \mathrm{Mo}$ predictions from the n-burst satisfy the requirements for the $\mathrm{SiC}-\mathrm{X}_{\mathrm{EM}}$ composition (see Table 1), ${ }^{92} \mathrm{Mo}$ and ${ }^{94} \mathrm{Mo}$ are ultimately synthesised in very small quantities $\left({ }^{92,94} \mathrm{Mo} /{ }^{97} \mathrm{Mo} \approx 10^{-3}\right)$. These points suggest that most of the p-only ${ }^{92} \mathrm{Mo}$ and ${ }^{94} \mathrm{Mo}$ present in SiC-X grains arise largely from the S.S. component and not the n-burst component. The same is true for ${ }^{96} \mathrm{Ru}$ and ${ }^{98} \mathrm{Ru}$ (see Table 1 ).

HEW component: Continuing with the analysis initially presented in $[19,28,29,34$, $36]$ we compute the Mo isotope ratios for cumulative entropy zones (from $S=10 \mathrm{k}_{\mathrm{B}}$ /baryon up to $\approx 270 \mathrm{k}_{\mathrm{B}}$ /baryon). For each cumulative entropy band, we mix the corresponding (cumulative) isotopic ratios with a S.S. composition to generate mixing lines. A 2D ordinary least squares fit of the mixing lines to the $\mathrm{SiC}-\mathrm{X}$ data in three-isotope space are used to constrain the optimum values of cumulative entropies ( $S$-range) to consider. The best fit for Mo isotopes is obtained for the case $Y_{e}=0.45$ with cumulative- $S \approx 94 \mathrm{k}_{\mathrm{B}} /$ baryon. Corresponding Mo isotope ratios are shown in Table 1 (column 3) and Fig. 3. These predictions - 'primary' HEW scenario - for ${ }^{92} \mathrm{Mo} /{ }^{97} \mathrm{Mo}$ and ${ }^{94} \mathrm{Mo} /{ }^{97} \mathrm{Mo}$ fall within the range of allowed $\mathrm{SiC}-\mathrm{X}_{\mathrm{EM}}$ compositions and are comparable to the 'secondary' n-burst predictions (Fig. 3). The ${ }^{95} \mathrm{Mo} /{ }^{97} \mathrm{Mo}$ value, which assumes close to S.S. values, is almost reproduced in our model (see Table 1), and the heavier isotopes ${ }^{98} \mathrm{Mo} /{ }^{97} \mathrm{Mo}$ and ${ }^{100} \mathrm{Mo} /{ }^{97} \mathrm{Mo}$ fall within a factor of two of the corresponding $\mathrm{SiC}-\mathrm{X}_{\mathrm{EM}}$ values. Finally, although ${ }^{96} \mathrm{Mo}$ is under produced $\left({ }^{96} \mathrm{Mo} /{ }^{97} \mathrm{Mo}=6.69 \times 10^{-5}\right)$, it does satisfy the $\mathrm{SiC}-\mathrm{X}_{\mathrm{EM}}$ constraint $\left({ }^{96} \mathrm{Mo} /{ }^{97} \mathrm{Mo}\right.$ $\leq 0.638$ ). A similar analysis for $\mathrm{Zr}$ reveals that the best fit between HEW theory and SiC-X data occur for slightly different conditions ( $Y_{e}=0.43$, cumulative- $S \approx 76 \mathrm{k}_{\mathrm{B}} /$ baryon), whereas preliminary analyses of $\mathrm{Ru}$ require slightly higher electron fractions and entropies ( $Y_{e} \approx 0.47$, cumulative- $S \approx 120 \mathrm{k}_{\mathrm{B}}$ /baryon). Overall, the best HEW model fits for $\mathrm{Zr}$, Mo and $\mathrm{Ru}$ occur for the low-S, CP-component of the HEW for a very small $Y_{e^{-}} S$ parameter window, indicating a consistent set of astrophysical conditions in the HEW of ccSNe.

TABLE 1: Mo isotope compositions of $\mathrm{SiC}-\mathrm{X}$ end-member and various nucleosynthetic processes.

\begin{tabular}{lcccccc}
\hline & ${\mathrm{SiC}-\mathrm{X}_{\mathrm{EM}}{ }^{\mathrm{a}}}$ & This work $^{\mathrm{b}}$ & n-burst & S.S.(s) & S.S.(r) $^{\mathrm{e}}$ & SN-Ia $^{\mathrm{f}}$ \\
\hline${ }^{\mathrm{i}} \mathrm{Mo} /{ }^{\mathrm{k}} \mathrm{Mo}:$ & & & & & & \\
${ }^{92} \mathrm{Mo} /{ }^{97} \mathrm{Mo}$ & $\leq 0.528$ & $2.74 \times 10^{-3}$ & $1.43 \times 10^{-3}$ & 0 & 0 & $\approx 27$ \\
${ }^{94} \mathrm{Mo} /{ }^{97} \mathrm{Mo}$ & $\leq 0.238$ & $1.73 \times 10^{-3}$ & $3.28 \times 10^{-3}$ & 0.013 & 0 & $\approx 18$ \\
${ }^{95} \mathrm{Mo} /{ }^{97} \mathrm{Mo}$ & $\approx 1.649$ & 2.321 & 1.540 & 1.801 & 1.382 & $\approx 5$ \\
${ }^{96} \mathrm{Mo} /{ }^{97} \mathrm{Mo}$ & $\leq 0.638$ & $6.69 \times 10^{-5}$ & 0.010 & 3.27 & 0 & $\approx 4$ \\
${ }^{98} \mathrm{Mo} /{ }^{97} \mathrm{Mo}$ & $\leq 1.133$ & 1.654 & 0.379 & 3.28 & 1.247 & $\approx 4$ \\
${ }^{100} \mathrm{Mo} /{ }^{97} \mathrm{Mo}$ & $\leq 0.390$ & 0.637 & 0.095 & 0.072 & 2.683 & $\approx 2$ \\
& & & & & & \\
${ }^{92} \mathrm{Mo} /{ }^{94} \mathrm{Mo}$ & $\geq 2.578$ & 1.587 & 0.437 & 0 & 0 & 1.527 \\
\hline
\end{tabular}

${ }^{\mathbf{a}} \mathrm{SiC}-\mathrm{X}_{\mathrm{EM}}$ limits. ${ }^{\mathbf{b}}$ Best case scenario for HEW nucleosynthesis occurs at $Y_{e}=0.45, S_{\text {cum }}=94$ (for Mo). ${ }^{\mathbf{c}}$ n-burst yields [22, 26]. ${ }^{\mathbf{d}}$ s-process yields [41]. ${ }^{\mathbf{e}}$-residuals [11, 41]. ${ }^{\mathbf{f}}$-process yields [43].

\section{Conclusions}


In the present paper we have shown selected results from an extension of our earlier largescale parameter study of the ccSN-HEW scenarios with a focus on its low-entropy $(S \leq 100$ - 150, $\left.Y_{n} / Y_{\text {seed }} \leq 1\right)$ charged-particle component. We confirm that for moderate electron abundances in the range $0.44 \leq Y_{e} \leq 0.49$, all p-, s- and r-process isotopes in the light transFe region between $\mathrm{Zn}(Z=30)$ and $\mathrm{Ru}(Z=44)$ can be co-produced in substantial yields. In this nucleosynthesis scenario, no initial S.S. or S.S.-modulated 'seed' composition (already containing the p-isotopes) is invoked as in e.g. [26, 43]. Hence, the low- $S$ component of the ccSN-HEW seems to be the main site of the primary production of the light p-isotopes, even resulting in a fair reproduction of the S.S. abundance ratio of the two most prominent p-isotopes ${ }^{92} \mathrm{Mo}$ and ${ }^{94} \mathrm{Mo}$.

Furthermore, particular attention has been directed towards the nucleosynthesis interpretation of the anomalous isotopic compositions of the stable isotopes of $\mathrm{Zr}$, Mo and $\mathrm{Ru}$ reported in SiC-X grains. Our geochemical analysis demonstrates that the measured grain data do not represent 'clean' nucleosynthesis signatures, but are a mixture of a minor, initially unknown exotic component with S.S. material. Therefore, discussions about the possible isotopic origins of these admixtures ( $\mathrm{SiC}-\mathrm{X}$ grains), e.g. as presented in [45] are questionable. Only after correcting for this S.S. 'contamination', by way of identifying the purest grain, may we proceed with explaining the isotopic compositions of these grains, which we do so in a consistent and realistic astrophysical way within a narrow band of $Y_{e}$ $S$ conditions in the charged-particle component of our ccSN-HEW. Finally we postulate that these S.S.-corrected SiC-X grain compositions are probably the only 'clean' signature of a standard ccSN-HEW scenario identified so far - without containing additional $r$ process admixtures from other explosive scenarios such as magnetorotational SN-Jets or neutron-star merger events usually observed in metal-poor halo stars.
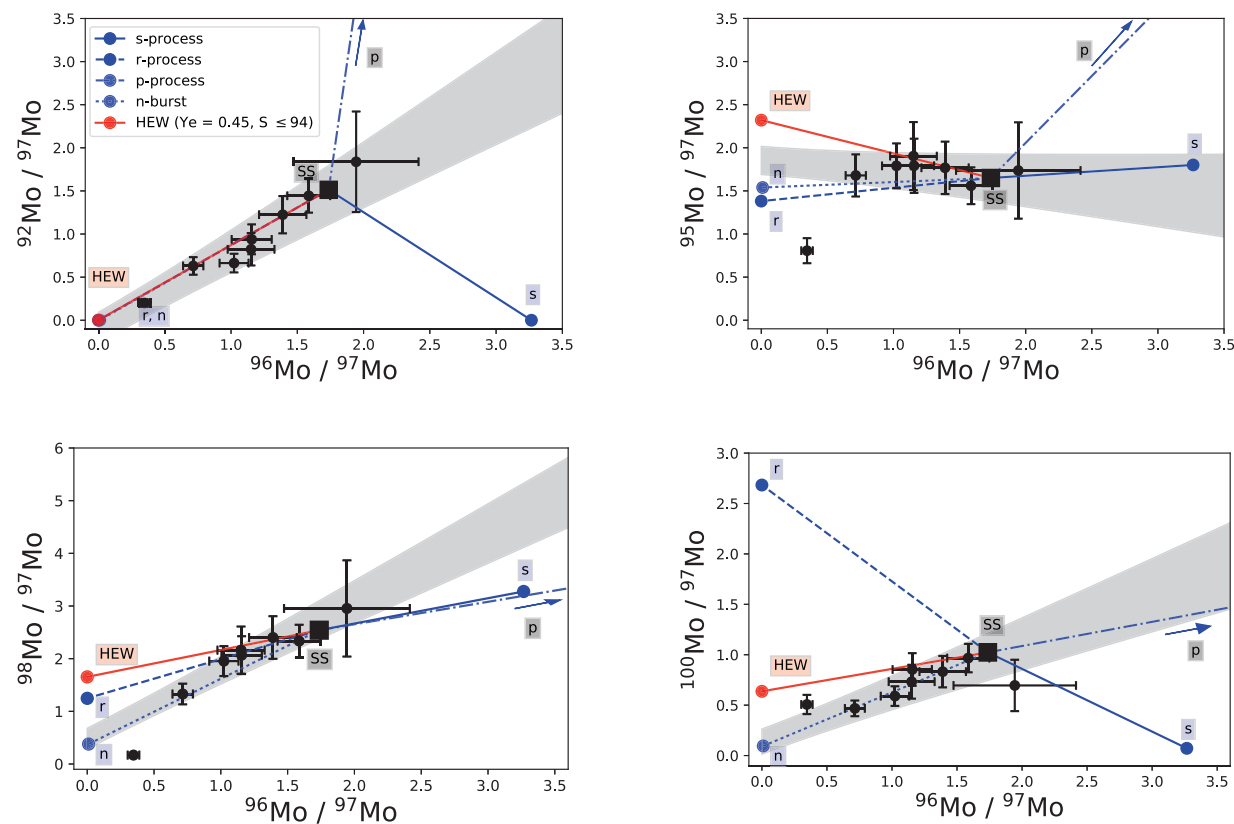

Fig. 3. Mo three-isotope plots for $\mathrm{SiC}-\mathrm{X}$ grains with mixing lines for various nucleosynthetic endmembers (see main text). Data uncertainties are $2 \sigma$. The error bands obtained from the 2D data fits (as in Fig. 2) are displayed as shaded areas. Filled circles indicate end-member compositions. 


\section{References}

1. E. M. Burbidge et al., Rev. Mod. Phys. 29, 547 (1957).

2. A. G. W. Cameron, PASP 69, 201 (1957).

3. C. D. Coryell, J. Chem. Education 38, 67 (1961).

4. M. Arnould, A\&A 46, 117 (1976).

5. S. E. Woosley, R. D. Hoffman, Ap. J. Suppl. 26, 285 (1978).

6. D. D. Clayton, Principles of Stellar Evolution and Nucleosynthesis (McGraw Hill, 1968).

7. F. Käppeler et al., Rep. Prog. Phys. 52, 945 (1989).

8. P. A. Seeger et al., Ap.J. Suppl. 11, 121 (1965).

9. W. Hillebrandt, Space Sci. Rev. 21, 639 (1978).

10. J. J. Cowan, F.-K. Thielemann, J. W. Truran, Phys. Rep. 208, 267 (1991).

11. K. Lodders, H. Palme, H.-P. Gail, "Abundances of the elements in the Solar System" in Solar System, Edited by J. E. Trümper (Elsevier, 2009).

12. C. Sneden, J. J. Cowan, R. Gallino, ARA\&A 46, 241 (2008).

13. I. U. Roederer et al., Ap.J. 724, 975 (2010).

14. V. Hill et al., A\&A 607, 91 (2017).

15. T. T. Hansen et al., Ap.J. 858, 92 (2018).

16. A. Frebel, T. C. Beers, Physics Today 72, 1, 30 (2018).

17. K.-L. Kratz et al., Ap.J. 403, 216 (1993).

18. K.-L. Kratz et al. Ap.J. 662, 39 (2007).

19. K.-L. Kratz et al. AIP Conference Proceedings 2076, 030002 (2019).

20. M. J. Pellin et al., LPSC XXXI, LPI 3131, 1917 (2000).

21. M. J. Pellin et al., LPSC XXXVII, LPI 37, 2041 (2006).

22. K. K. Marhas, P. Hoppe, U. Ott, MAPS 42, 1077 (2007).

23. W. Akram et al., Ap.J. 777, 169 (2013).

24. U. Ott, "Isotope Variations in the Solar System: Supernova Fingerprints" in Handbook of Supernovae, edited by A. W. Alsabi and P. Murdin (Springer International Publishing, Switzerland, 2016).

25. M. Howard et al., Meteoritics 27, 404 (1992).

26. B. S. Meyer, D. D. Clayton, L.-S. The, Ap.J. 540, L52 (2000).

27. B. S. Meyer (private communication).

28. K. Farouqi, K.-L. Kratz, B. Pfeiffer, PASA 26, 194 (2009).

29. K. Farouqi et al., XI Symposium on Nuclei in the Cosmos, PoS 081 (2010).

30. S. E. Woosley et al., Ap.J. 433, 229 (1994).

31. K. Takahashi, J. Witti, H.-T. Janka, A\&A 286, 857 (1994).

32. K.-L. Kratz et al., NewAR 52, 390 (2008).

33. K.-L. Kratz, K. Farouqi, P. Möller, Ap.J. 792, 6 (2014).

34. K. Farouqi et al., Ap.J. 694, L49 (2009).

35. C. Freiburghaus et al., Ap.J. 516, 381 (1999).

36. K. Farouqi et al., Ap.J. 712, 1359 (2010).

37. O. Hallmann et al., $76^{\text {th }}$ Met. Soc. Meeting, 5082 (2013).

38. E. Zinner, "Presolar grains", in Treatise on Geochemistry, $2^{\text {nd }}$ Edition, edited by H. D. Holland and K. K. Turekian (Elsevier, 2014), pp181-213.

39. E. Zinner, "Presolar material in meteorites: an overview", in Astrophysical Implications of the Laboratory Study of Presolar Materials, edited by T. J. Bernatowicz and E. Zinner (AIP, New York), pp3-26.

40. C. Arlandini et al., Ap.J. 525, 886 (1999).

41. S. Bisterzo et al., Mon. Not. R. Astron. Soc. 418, 284 (2011).

42. S. Cristallo et al., J. Phys.: Conf. Ser. 665, 012019 (2016).

43. C. Travaglio et al., Ap.J. 739, 93 (2011).

44. S. Wanajo et al., Ap.J. 695, 208 (2009).

45. M. Eichler et al., arXiv: 1708.08393 (2017). 